\title{
Electrochemical processes in all-solid-state Li-S batteries studied by electrochemical impedance spectroscopy
}

\author{
Bo Fan ${ }^{\mathrm{a}, \mathrm{b}}$, Zebo Guan ${ }^{\mathrm{a}}$, Hongjiao Wang ${ }^{\mathrm{a}, \mathrm{c}}$, Lilin Wu ${ }^{\mathrm{d}}$, Wenzhi Li ${ }^{\mathrm{e}}$, Shibang Zhang ${ }^{\mathrm{a}}$, \\ Bai Xue ${ }^{a^{*}}$ \\ a Shenzhen Key Laboratory of Advanced Thin Films and Applications, College of Physics and Optoelectronic \\ Engineering, Shenzhen University, 518060 Shenzhen, China \\ b State Key Lab of Silicon Materials, Zhejiang University, Hangzhou 310027, China \\ ${ }^{\mathbf{c}}$ Laboratory of Glasses and Ceramics, Institute of Chemical Science, University of Rennes 1, Rennes 35042, \\ France \\ d College of Chemistry and Environmental Engineering, Shenzhen University, 518060 Shenzhen, China \\ e College of Materials Science and Engineering, Shenzhen University, 518060 Shenzhen, China
}

\begin{abstract}
Understanding the electrochemical processes in all-solid-state lithium-sulfur batteries is essential for designing high-performance devices. Here, the evolution of the electrochemical impedance spectra of an all-solid-state lithium-sulfur battery during one charge/discharge cycle was quantitatively investigated with a reasonably established equivalent circuit. The impedance spectra could be decomposed into several parts as follows: the bulk resistance of solid electrolyte and current collector at highest frequency, three semicircles from high to low frequency corresponding to the dynamic processes at different interfaces and a $45^{\circ}$ straight line followed by an inclined small tail at low frequency. It is found that the middle frequency semicircle, corresponding to charge transfer at the cathode, varied obviously during the charge/discharge cycle. Moreover, the $45^{\circ}$ straight line, which is attributed to the Li diffusion in the cathode material $\mathrm{Li}_{\mathrm{x}} \mathrm{S}$ based on qualitative analysis, dominates the impedance spectra, revealing that the main kinetics-limiting factor is the Li diffusion in the active materials. Finally, an electrochemical model of the battery is proposed which provides insight for the designing of highperformance all-solid-state lithium-sulfur batteries.
\end{abstract}

\section{ACCEPTED MANUSCRIPT}


Keywords: electrochemical impedance spectroscopy; all-solid-state battery; lithium-sulfur battery; mechanism model;

* Corresponding author. Tel: +86 (0)755 26534995

E-mail address: baixue@ szu.edu.cn

\section{Introduction}

Society's requirements of new energy source technologies such as electrical vehicles and new energy plants arise the enthusiasm to find novel secondary batteries with high energy density, long cycle life and high safety. Due to the high energy density (2600 $\left.\mathrm{KW} \cdot \mathrm{h}^{-1}\right)$ and high theoretical specific capacity $\left(1675 \mathrm{mAh} \cdot \mathrm{g}^{-1}\right)$, lithium-sulfur batteries are considered as one of the best candidates for energy storage system in the future. Additionally, the cathode material of lithium-sulfur batteries, elemental S, is nontoxic, low-cost and easy access.[1-3] However, there are still many issues needed to be addressed, including polysulfide shuttling, lithium-dendrite growth, poor thermal stability and flammability, and these problems lead to short lifetime and capacity fading of the batteries.[4] Recently, the all-solid-state lithium-sulfur batteries (ASSLSBs) using inorganic solid electrolyte instead of liquid-based electrolyte have attracted the attention of researchers because not only the ASSLSBs overcome the polysulfideshuttling, but also effectively suppress the lithium-dendrites due to the advantages of strong rigidity.[3] However, the development of ASSLSBs has been limited by other difficulties, including poor ionic/electronic conductivity between solid-solid interfaces, slow reaction kinetics, large volume expansion of electrodes and low utilization of

\section{ACCEPTED MANUSCRIPT}


active materials during charge/discharge processes.[1,3] These drawbacks will deteriorate the performance of ASSLSBs.

In order to solve these problems, it is important to understand the electrochemical processes of ASSLSBs. So far, different methods have been taken to explore the reaction mechanism of ASSLSBs. For instance, Hyea et al. used in-situ transmission electron microscope (TEM) to study the lithiation processes of sulfur in carbon nanotubes and observed that $\mathrm{S}$ directly transfer to $\mathrm{Li}_{2} \mathrm{~S}$ without generating polysulfides.[5] Yang et al. also monitored the lithiation processes of sulfur on carbon film by in-situ TEM, and found the phase separation of $\mathrm{S} / \mathrm{Li}_{2} \mathrm{~S}$ at nanoscale which facilitates the formation of electronic channels at the $\mathrm{S} / \mathrm{Li} 2 \mathrm{~S}$ interfaces.[6] Wenzel et al. obtained the detailed information on the reaction at the $\mathrm{Li} / \mathrm{Li}_{10} \mathrm{GeP}_{2} \mathrm{~S}_{12}$ interface by XPS, illustrating that the decomposition of the solid electrolyte into $\mathrm{Li}_{3} \mathrm{P}, \mathrm{LiS}$ and LiGe alloy leads to the continuous growth of the interfacial resistance.[7]

Besides microscopic characterization technologies, electrochemical impedance spectroscopy (EIS) is another powerful tool to study the electrochemical processes of batteries. In the field of the conventional liquid lithium-sulfur batteries, EIS has been widely used to clarify the reaction mechanism and monitor the change of resistance at different interfaces. Deng et al. proposed an equivalent circuit model for the liquid lithium-sulfur battery based on the impedance spectra at various temperatures and discharge depths, which provides quantitative monitoring of the evolution of the interphase contact resistance and the charge-transfer resistance during cycling.[8] Canas et al. elucidated that charge transfer at the cathode/electrolyte interface

\section{ACCEPTED MANUSCRIPT}


influences the capacity of liquid lithium-sulfur batteries by studying EIS at different depths of charge/discharge.[9]

Although EIS is also widely used in the field of ASSLSBs, $[2,10,11]$ only a few of them applied detailed analysis of the spectra to deeply discuss the electrochemical reaction processes of ASSLSBs. As one of the limited number of examples, Wang et al. established a transmission line model to describe the EIS of an all-solid-state Li-S primary battery, and proposed that the kinetics of electrode during discharge is primarily determined by ionic migration at the electrode level according to their quantitative analysis.[12] However this work encountered the problem of the overestimation of charge transfer resistance. In this paper, the evolution of impedance spectra of an ASSLSB is monitored during charge/discharge cycling. An equivalent circuit is established to describe the recorded impedance spectra so that the key parameters associated with different electrochemical processes inside the battery can be determined by EIS fitting. By analyzing how these parameters change at different depths of charge/discharge, an electrochemical model of the battery is proposed which provides insight for the designing of high-performance ASSLSBs.

\section{Experimental procedures}

The starting materials include $\mathrm{Li}_{2} \mathrm{~S}$ (99.9\%, Alfa Aesar), $\mathrm{P}_{2} \mathrm{~S}_{5}$ (99\%, Qingdao Yurui Ltd.), sublimated sulfur (99.5\%, Alfa Aesar), Ketjen Black (KB, ECP600JD), anhydrous tetrahydrofuran (THF, $\geq 99.9 \%$, Aladdin) and anhydrous acetonitrile (ACN, 99.8\%, Aladdin).

$\mathrm{Li}_{7} \mathrm{P}_{3} \mathrm{~S}_{11}$ glass-ceramic electrolyte was obtained by planetary ball milling and heat

\section{ACCEPTED MANUSCRIPT}


treatment. A mixture of $\mathrm{Li}_{2} \mathrm{~S}$ and $\mathrm{P}_{2} \mathrm{~S}_{5}$ (molar ratio 7:3) was putted into tungsten carbonide jar. Then, ball milling was conducted at $450 \mathrm{rpm}$ using planetary ball mill apparatus (Pulverisette 7, Fritsch GmbH, Germany) lasting $70 \mathrm{~h}$. All of the operations were conducted in an argon-filled glovebox $\left(\mathrm{O}_{2}<0.5 \mathrm{ppm}, \mathrm{H}_{2} \mathrm{O}<0.5 \mathrm{ppm}\right)$. After ball milling, the resultant $\mathrm{Li}_{2} \mathrm{~S}-\mathrm{P}_{2} \mathrm{~S}_{5}$ powder was sealed in a silica glass tube under vacuum and heat-treated at $260{ }^{\circ} \mathrm{C}$ for $1 \mathrm{~h}$ to obtain $\mathrm{Li}_{7} \mathrm{P}_{3} \mathrm{~S}_{11}$ glass-ceramic powder.

The composite cathode was prepared by liquid-phase method. Firstly, $\beta$-Li ${ }_{3} \mathrm{PS}_{4} / \mathrm{S}$ composite was prepared by dual-solution synthesis as described in our previous study.[13] Briefly, tetrahydrofuran solution of $\mathrm{Li}_{2} \mathrm{~S}_{6}$ and acetonitrile solution of $\mathrm{Li}_{2} \mathrm{~S} \cdot \mathrm{P}_{2} \mathrm{~S}_{5}$ were prepared by stirring the raw materials $\left(\mathrm{Li}_{2} \mathrm{~S}\right.$ and $\mathrm{S}$ for $\mathrm{Li}_{2} \mathrm{~S}_{6}, \mathrm{Li}_{2} \mathrm{~S}$ and $\mathrm{P}_{2} \mathrm{~S}_{5}$ for $\left.\mathrm{Li}_{2} \mathrm{~S} \cdot \mathrm{P}_{2} \mathrm{~S}_{5}\right)$ in the solvents, and then two solutions were mixed. The precursor solution was dried at $60{ }^{\circ} \mathrm{C}$ to remove the excess solvent and further heat-treated at $230{ }^{\circ} \mathrm{C}$ under argon for $1 \mathrm{~h}$ to obtain the products. Finally, a mixture of $\beta$-Li3 $\mathrm{PS}_{4} / \mathrm{S}$, $\mathrm{Li}_{7} \mathrm{P}_{3} \mathrm{~S}_{11}$ and $\mathrm{KB}$ (mass ratio 5:3:2) was ball-milled at $450 \mathrm{rpm}$ for $6 \mathrm{~h}$ to obtain the composite cathode.

The phase structure was tested by the X-ray diffraction (XRD, D8-Advanced, Bruker AXS GmbH, Germany) using $\mathrm{Cu}-\mathrm{Ka}$ radiation $(\lambda=1.504 \AA$ ). To avoid the moisture, the samples were mounted in the sample holder and sealed by polyimide cover films in the glovebox prior to XRD characterization. For the measurement of ionic conductivity, the samples were sandwiched between two layers of acetylene black powder and coldpressed into pellets (15 mm diameter and $\sim 1 \mathrm{~mm}$ thickness) with $330 \mathrm{MPa}$ pressure. AC impedance spectra were recorded by used a frequency response analyzer (Solartron

\section{ACCEPTED MANUSCRIPT}


1260A, Solartron Analytical Inc., UK) to determine the ionic conductivity.

All-solid-state lithium-sulfur batteries were assembled as follow. Firstly, $300 \mathrm{mg}$ $\mathrm{Li}_{7} \mathrm{P}_{3} \mathrm{~S}_{11}$ powder was cold-pressed into pellet under a pressure of $80 \mathrm{MPa}$ for $1 \mathrm{~min}$. Then, $4 \mathrm{mg}$ powder of composite cathode was spread on one side of the pellet and coldpressed under a pressure of $330 \mathrm{MPa}$ for $10 \mathrm{~min}$, and a Li foil (diameter: $13 \mathrm{~mm}$ ) was attached to the other side of the pellet. Finally, the pellet was packaged by a swagelok mold. The battery assembling was conducted in an argon-filled glovebox $\left(\mathrm{O}_{2}<0.5 \mathrm{ppm}\right.$, $\mathrm{H}_{2} \mathrm{O}<0.5 \mathrm{ppm}$ ). For comparison, a typical liquid lithium-sulfur battery using S-C cathode and 1 M LiTFSI DME/DOL (50-50 vol\%)-1\% $\mathrm{LiNO}_{3}$ electrolyte, was also assembled according to the reported method. $[14,15]$

The charge and discharge curves of the all-solid-state lithium-sulfur battery were recorded by a battery tester (LAND CTZ001A) with a constant current of $0.1 \mathrm{~mA}$ and a voltage range of 1.5-3.0 V. The EIS measurements were performed at an electrochemical workstation (CHI660E, CH Instruments Ins., Shanghai, China). The frequency range was $10 \mathrm{mHz}$ to $1 \mathrm{MHz}$ and the amplitude of AC signal was $5 \mathrm{mV}$. After testing, the impedance spectra were fitted by using Zview software.

\section{Results and discussions}

Figure 1(a) shows the XRD pattern of the $\beta$ - $\mathrm{Li}_{3} \mathrm{PS}_{4} / \mathrm{S}$ composite. The characteristic diffraction peaks of elemental S and $\beta$-Li3 $\mathrm{PS}_{4}$ can be observed. It is well-known that $\mathrm{S}$ is usually used as active materials and $\beta-\mathrm{Li}_{3} \mathrm{PS}_{4}$ is an electrolyte with good Li-ion conductivity. The impedance spectrum of the composite is shown in Figure 1(b). According to the equation $\sigma=l /(R A)[16,17]$, where $l, A$ and $R$ are the thickness, the

\section{ACCEPTED MANUSCRIPT}


surface area and the resistance (the value of intercept of the low-frequency tail and real axis) of the pellet respectively, the ion conductivity $\sigma$ of the composite is calculated to be $3.0 \times 10^{-6} \mathrm{~S} \cdot \mathrm{cm}^{-1}$. For comparison, the impedance spectrum of pure sulfur was tested and shown in the Figure 1(c). It behaves as a capacitor showing that the resistance of pure sulfur is extremely high. According to the literature, the electrical conductivity of pure sulfur is only $1.64 \times 10^{-30} \mathrm{~S} \cdot \mathrm{cm}^{-1}[18] . \beta-\mathrm{Li}_{3} \mathrm{PS}_{4} / \mathrm{S}$ composite shows much higher ionic conductivity than pure sulfur, that is benefited from the combination with $\beta$ $\mathrm{Li}_{3} \mathrm{PS}_{4}\left(1.64 \times 10^{-4} \mathrm{~S} \cdot \mathrm{cm}^{-1}\right)[19]$. This $\beta-\mathrm{Li}_{3} \mathrm{PS}_{4} / \mathrm{S}$ composite replaces pure sulfur as the cathode material of the batteries, and its significantly improved ionic conductivity will promote the reaction kinetics of the cathode.
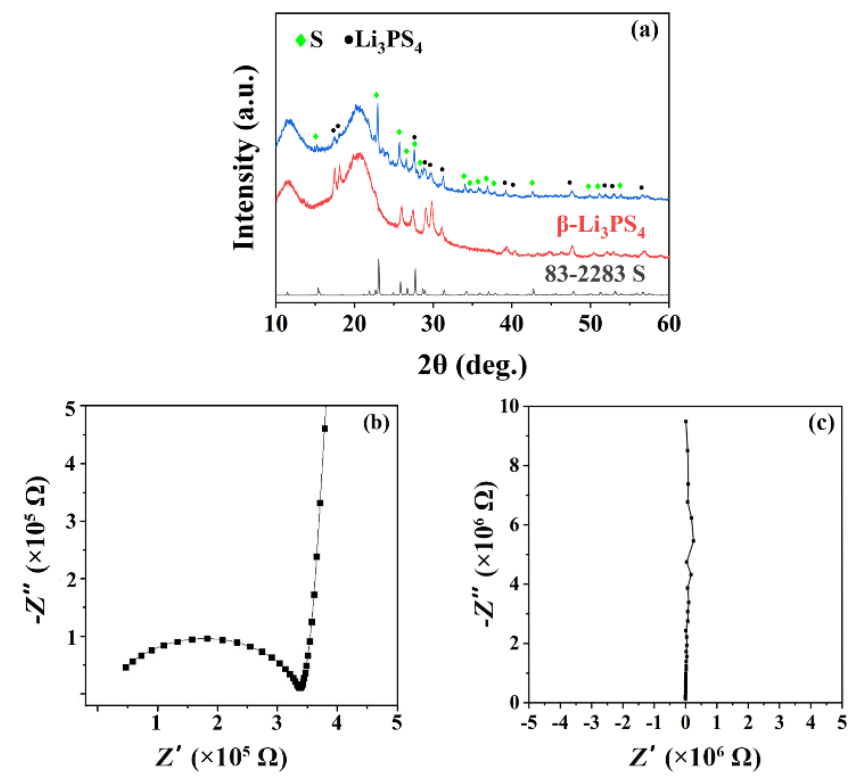

Figure 1. (a) XRD pattern and impedance spectrum of (b) the $\beta-\mathrm{Li}_{3} \mathrm{PS}_{4} / \mathrm{S}$ composite and (c) pure sulfur.

Figure 2(a) presents the discharge and charge curves of the ASSLSB under stable operation. For comparison, the typical liquid lithium-sulfur battery using ether-based 
liquid electrolyte, as shown in Figure 2(b), has two voltage plateaus during discharge. The upper plateau corresponds to the conversion from sulfur to soluble high-order lithium polysulfides, and the lower plateau is related to the further reduction of lithium polysulfides to $\mathrm{Li}_{2} \mathrm{~S} / \mathrm{Li}_{2} \mathrm{~S}$.[20] Differently, only one plateau appears in the discharge curve of the ASSLSB, indicating a direct conversion from elemental S to the reduction products $\mathrm{Li}_{\mathrm{x}} \mathrm{S}$.[ 5, 21-23] Reverse process occurs during charging of the battery, where $\mathrm{Li}_{\mathrm{x}} \mathrm{S}$ converts to elemental $\mathrm{S}$ again. The absent of soluble polysulfides in ASSLSBs solves the problem of polysulfide shuttling in liquid lithium-sulfur batteries. In order to test the impedance spectra at different depths of charge and discharge, the battery is stopped at a series of preset voltages and rests until the open circuit voltage becomes stable. 

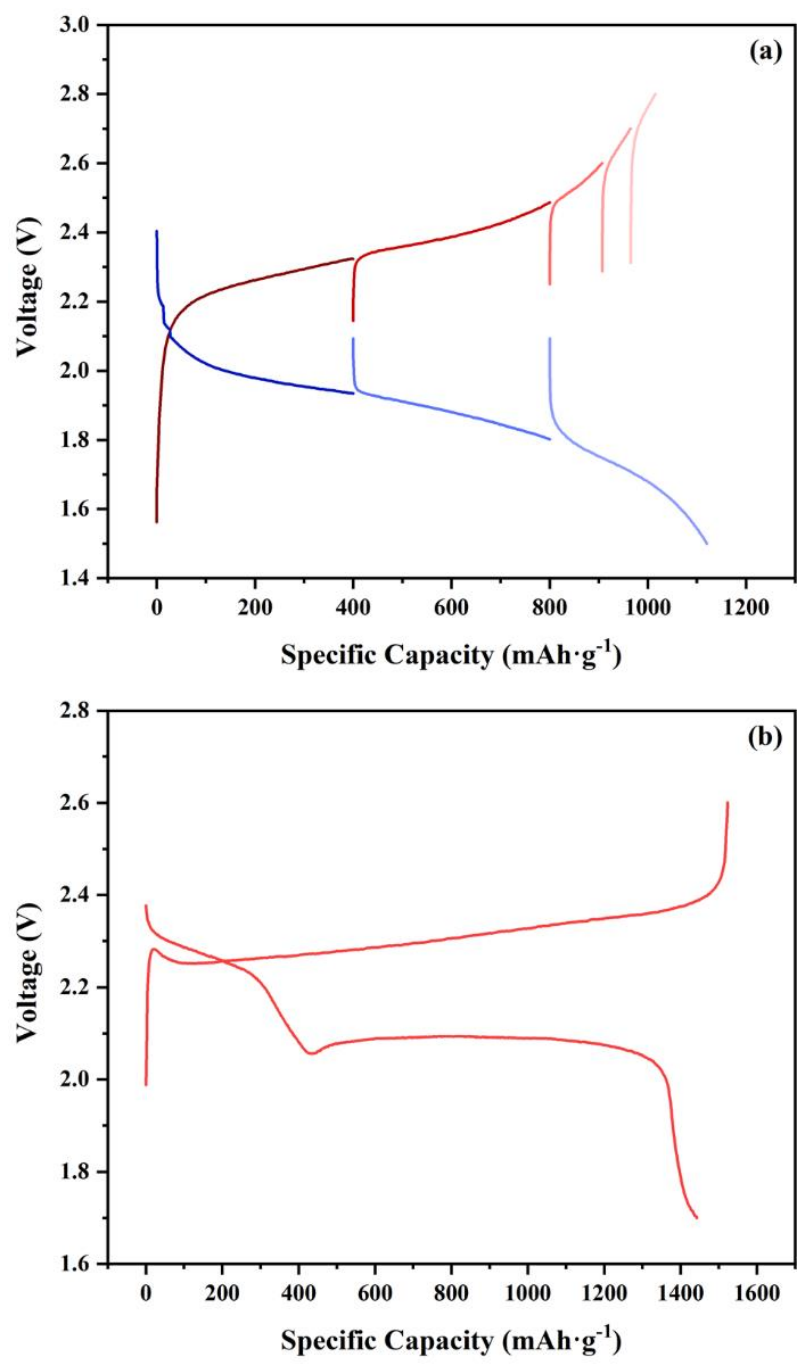

Figure 2. Charge and discharge curves of the (a) ASSLSB and (b) typical liquid lithium-sulfur battery.

Figure 3 illustrates the Nyquist plots of the battery at different depths of discharge and charge. Typically, the impedance spectrum is composed of three semicircles, which locate at high frequency, middle frequency and low frequency respectively, as shown by the schematic diagram in Figure 4. Additionally, there is a tail with a slope of about $45^{\circ}$ at the low-frequency end. Similar characteristics of the impedance spectra have been reported for other types of all-solid-state batteries. Larfaillou et al. illustrated that the three semicircles from high to low frequency correspond to solid electrolyte

\section{ACCEPTED MANUSCRIPT}


resistance, interfacial resistance of cathode/electrolyte and anode/electrolyte respectively, while the $45^{\circ}$ slope represents the Warburg diffusion behavior.[24] Zhang et al. also found three semicircles in all-solid-state lithium-ion batteries.[25] Notably, the slope of the tail at the lowest frequency changes at different depths of discharge and charge. This could be attributed to the manifestation of the capacitive impedance associated with the intercalation capacitance of the battery.[ 26,27$]$
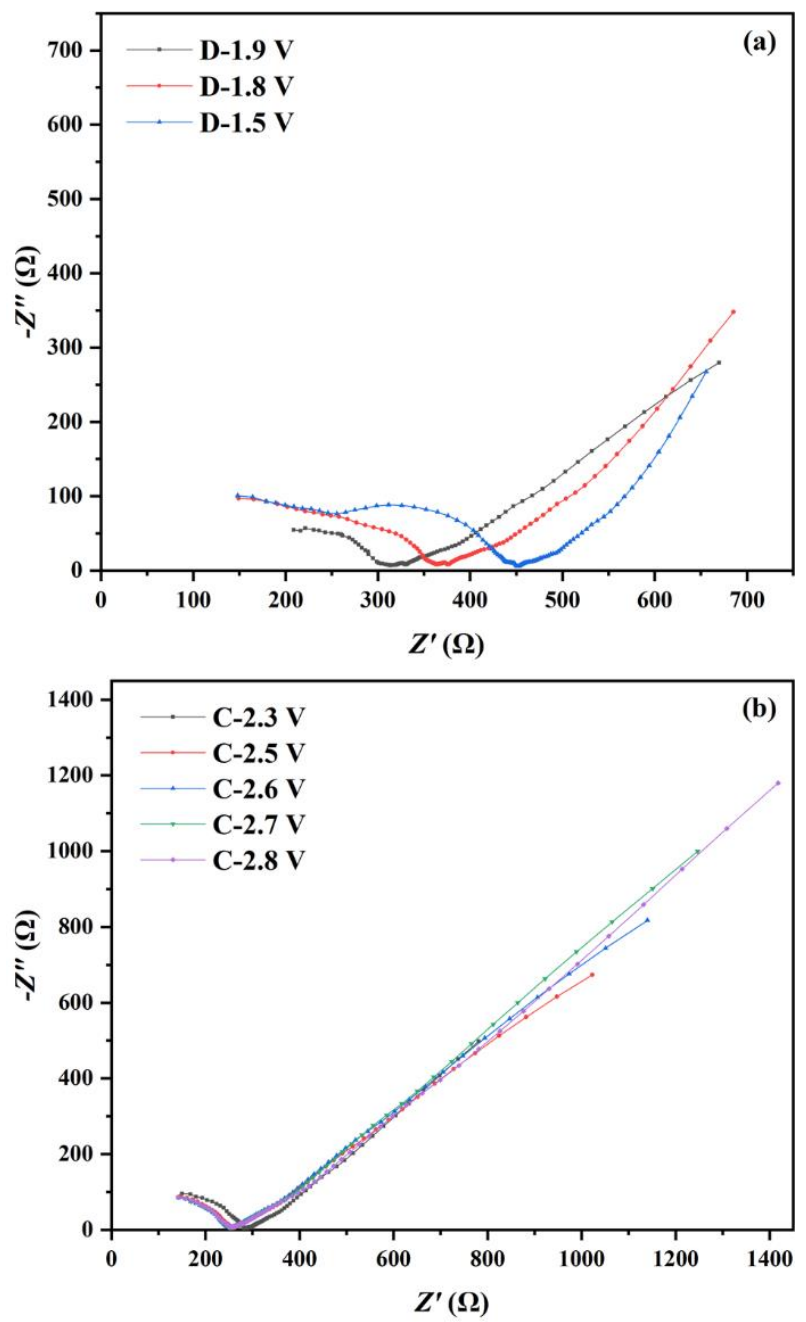

Figure 3. Nyquist plots of the ASSLSB at different depths of (a) discharge and (b) charge. 


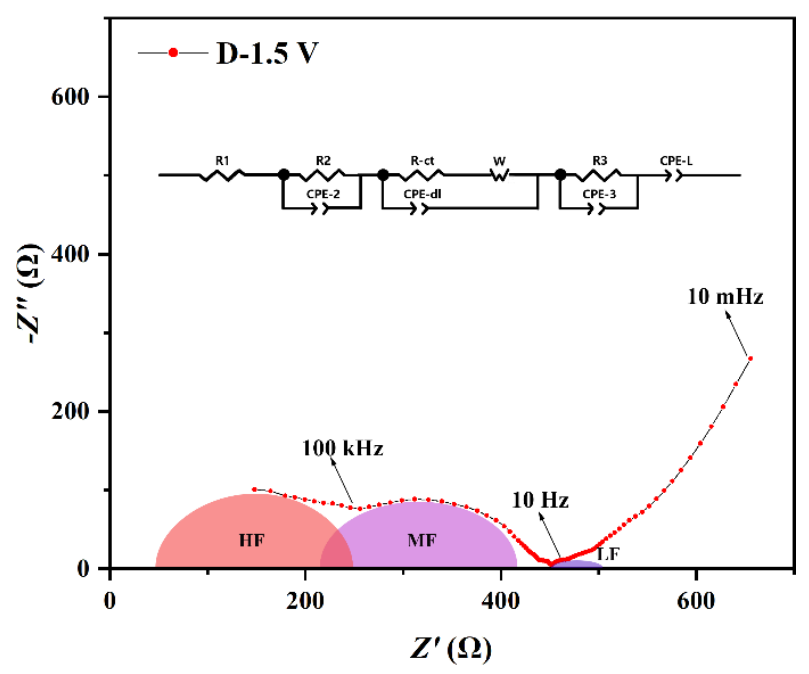

Figure 4. Schematic diagram of the EIS semicircles in different frequency ranges. The insert shows the proposed equivalent circuit for the EIS of the ASSLSB.

According to the characteristics of the impedance spectra, an equivalent circuit is assigned to describe the impedance spectra of the studied ASSLSB, as shown in the inset of Figure 4. Usually, a pure resistor in parallel with a capacitive element is used to describe a semicircle in Nyquist plots. Here CPEs (constant phase elements) are used instead of capacitors to describe the non-ideal behavior of the system resulting from rough or porous surface of materials.[8] The impedance spectra at different depths of discharge/charge are fitted based on the equivalent circuit. The fitted curves are in good agreement with the experimental spectra as shown in Figure 5 and Figure 6. The fitting parameters are summarized in Table 1 and Table 2. The resistances are further specified to the sample area $\left(1.33 \mathrm{~cm}^{2}\right)$ in $\Omega \cdot \mathrm{cm}^{2}$ and illustrated in Figure 7 . 


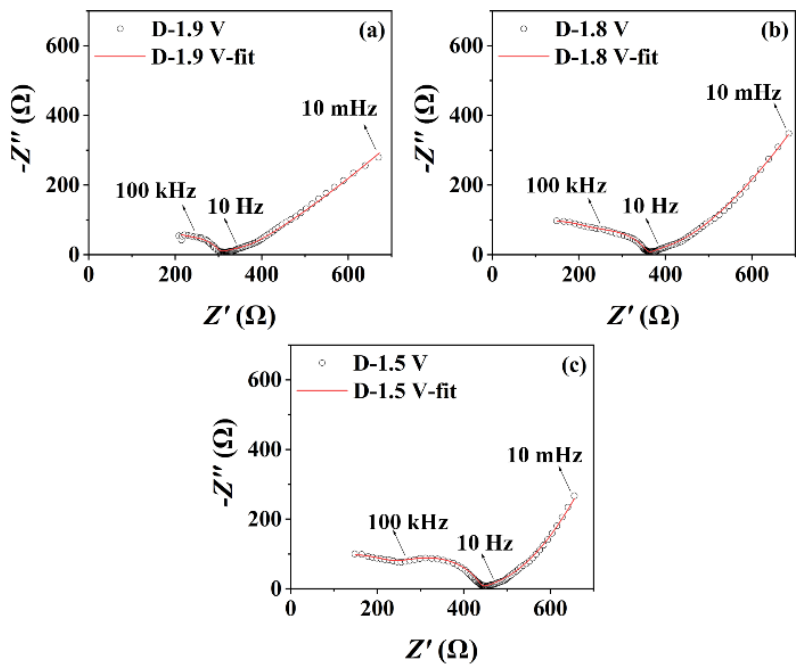

Figure 5. Fitted plots at different depths of discharge: (a) D-1.9 V (b) D-1.8 V (c) D-1.5 V.

Table 1. Fitting parameters of the EISs at different depths of discharge (DOD).

\begin{tabular}{|c|c|c|c|c|}
\hline DOD & & $1.9 \mathrm{~V}$ & $1.8 \mathrm{~V}$ & $1.5 \mathrm{~V}$ \\
\hline$R_{1} / \Omega$ & & 23.6 & 25.4 & 22.0 \\
\hline$R_{2} / \Omega$ & & 195.3 & 194.0 & 211.7 \\
\hline \multirow[t]{2}{*}{$\mathrm{CPE}_{2}$} & $T / \mathrm{F} \cdot \mathrm{s}^{\mathrm{P}-1}$ & $9.95 \mathrm{E}-09$ & 4.53E-09 & $6.47 \mathrm{E}-09$ \\
\hline & $P$ & 0.8145 & 0.8865 & 0.8613 \\
\hline$R_{\mathrm{CT}} / \Omega$ & & 78.0 & 136.6 & 202.8 \\
\hline \multirow[t]{2}{*}{$\mathrm{CPE}_{\mathrm{dl}}$} & $T / \mathrm{F} \cdot \mathrm{s}^{\mathrm{P}-1}$ & $1.89 \mathrm{E}-07$ & $2.45 \mathrm{E}-07$ & $2.09 \mathrm{E}-07$ \\
\hline & $P$ & 0.8488 & 0.7916 & 0.8219 \\
\hline$R_{3} / \Omega$ & & 67.3 & 87.5 & 88.3 \\
\hline \multirow[t]{2}{*}{$\mathrm{CPE}_{3}$} & $T / \mathrm{F} \cdot \mathrm{s}^{\mathrm{P}-1}$ & $1.95 \mathrm{E}-03$ & $4.45 \mathrm{E}-03$ & 8.81E-03 \\
\hline & $P$ & 0.3847 & 0.3800 & 0.3194 \\
\hline \multirow[t]{2}{*}{$\mathrm{W}$} & $T / \mathrm{F} \cdot \mathrm{s}^{\mathrm{P}-1}$ & $8.51 \mathrm{E}-03$ & $1.14 \mathrm{E}-02$ & $1.87 \mathrm{E}-02$ \\
\hline & $P$ & 0.4430 & 0.4983 & 0.4950 \\
\hline \multirow[t]{2}{*}{$\mathrm{CPE}_{\mathrm{L}}$} & $T / \mathrm{F} \cdot \mathrm{s}^{\mathrm{P}-1}$ & $4.15 \mathrm{E}-01$ & $1.66 \mathrm{E}-01$ & $1.49 \mathrm{E}-01$ \\
\hline & $P$ & 0.9545 & 0.9863 & 0.9898 \\
\hline
\end{tabular}

The impedance of CPE is defined as $Z_{C P E}=\frac{1}{T(i \omega)^{P}}$. CPE turns to capacitance when $P=1$. The impedance of Warburg element is defined as $Z_{W}=\frac{1}{T(i \omega)^{P}}$. For ideal Warburg element $P=0.5$, which can be slightly lower than 0.5 due to material inhomogeneity.

\section{ACCEPTED MANUSCRIPT}



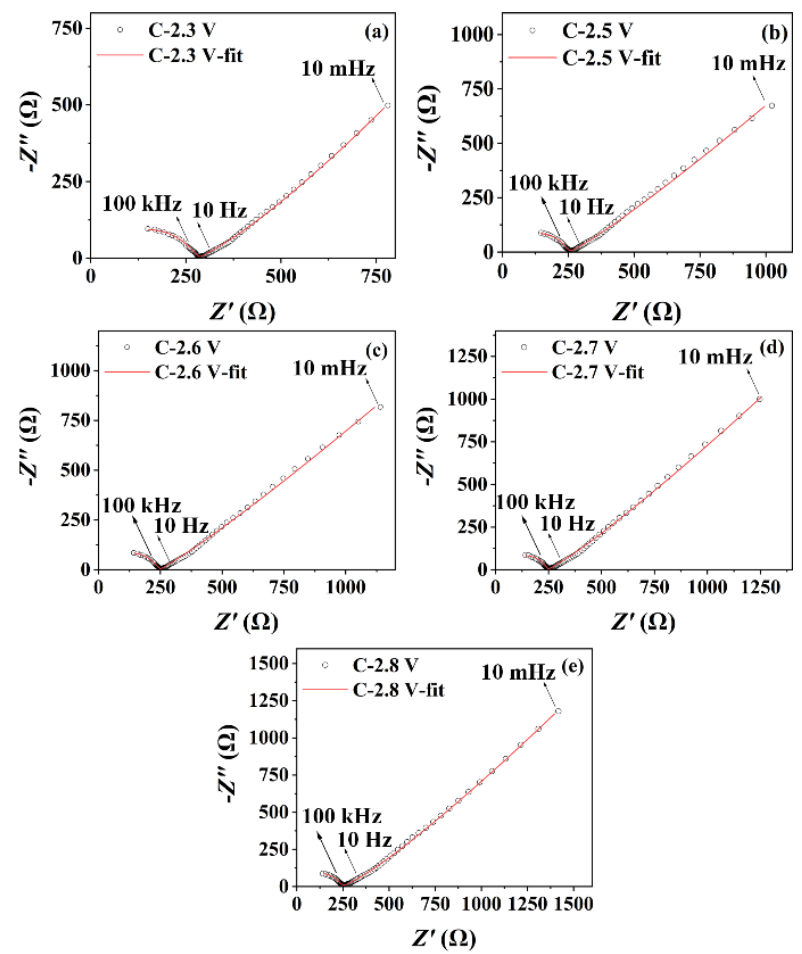

Figure 6. Fitted plots at different depths of charge: (a) C-2.3 V (b) C-2.5 V (c) C-2.6 V

$$
\text { (d) C-2.7 V (e) C-2.8 V. }
$$

Table 2. Fitting parameters of the EISs at different depths of charge (DOC).

\begin{tabular}{lllllll}
\hline $\mathrm{DOC}$ & & $2.3 \mathrm{~V}$ & $2.5 \mathrm{~V}$ & $2.6 \mathrm{~V}$ & $2.7 \mathrm{~V}$ & $2.8 \mathrm{~V}$ \\
\hline$R_{1} / \Omega$ & & 25.0 & 24.2 & 21.3 & 22.6 & 21.7 \\
$R_{2} / \Omega$ & & 202.0 & 203.2 & 199.7 & 200.6 & 206.5 \\
$\mathrm{CPE}_{2}$ & $T / \mathrm{F} \cdot \mathrm{s}^{\mathrm{P}-1}$ & $7.24 \mathrm{E}-09$ & $6.24 \mathrm{E}-09$ & $7.44 \mathrm{E}-09$ & $7.15 \mathrm{E}-09$ & $5.58 \mathrm{E}-09$ \\
& $P$ & 0.8625 & 0.8666 & 0.8540 & 0.8589 & 0.8721 \\
& & 56.0 & 25.5 & 25.9 & 22.9 & 19.8 \\
$R_{\mathrm{CT}} / \Omega$ & & $2.41 \mathrm{E}-06$ & $4.06 \mathrm{E}-06$ & $3.24 \mathrm{E}-06$ & $2.33 \mathrm{E}-06$ & $6.20 \mathrm{E}-06$ \\
$\mathrm{CPE}$ & $T / \mathrm{F} \cdot \mathrm{s}^{\mathrm{P}-1}$ & 0.6233 & 0.6785 & 0.8536 & 0.7102 & 0.7004 \\
& $P$ & 72.4 & 71.7 & 82.9 & 83.6 & 93.6 \\
$R_{3} / \Omega$ & & $9.00 \mathrm{E}-03$ & $9.21 \mathrm{E}-03$ & $8.95 \mathrm{E}-03$ & $6.01 \mathrm{E}-03$ & $3.28 \mathrm{E}-03$ \\
$\mathrm{CPE}$ & $T / \mathrm{F} \cdot s^{\mathrm{P}-1}$ & 0.3621 & 0.3055 & 0.3527 & 0.3636 & 0.3648 \\
& $P$ & $6.59 \mathrm{E}-03$ & $3.96 \mathrm{E}-03$ & $3.45 \mathrm{E}-03$ & $3.02 \mathrm{E}-03$ & $2.63 \mathrm{E}-03$
\end{tabular}




\begin{tabular}{lllllll} 
& $P$ & 0.4989 & 0.4703 & 0.4852 & 0.4999 & 0.4919 \\
$\mathrm{CPE}_{\mathrm{L}}$ & $T / \mathrm{F} \cdot \mathrm{s}^{\mathrm{P}-1}$ & $2.74 \mathrm{E}-01$ & $3.87 \mathrm{E}-01$ & $3.67 \mathrm{E}-01$ & $2.25 \mathrm{E}-01$ & $1.30 \mathrm{E}-01$ \\
& $P$ & 0.9953 & 0.9905 & 0.9875 & 0.9951 & 0.9941 \\
\hline
\end{tabular}

The intercept of the spectra with the real-axis at high frequency end is represented by a pure resistor $R_{1}$ of about $30 \Omega \cdot \mathrm{cm}^{2}$, and is almost unchanged at different depths of discharge/charge as illustrated in the Figure 7. $R_{1}$ approaches the resistance of the solidelectrolyte separator made of $\mathrm{Li}_{7} \mathrm{P}_{3} \mathrm{~S}_{11}$, thus corresponds to the bulk resistance of the solid-electrolyte and the current collector of the battery. The high-frequency semicircle described by the parallel of $R_{2}$ and $C P E_{2}$ also changes little during one charge/discharge cycle. According to the literature[28-30], the high-frequency semicircle corresponds to the resistance and the related capacitance of the electrode/electrolyte interphase layers. Therefore, it can be concluded that the electrode/electrolyte interfaces are stable against the variation of electrochemical state of the electrodes during one cycle. 

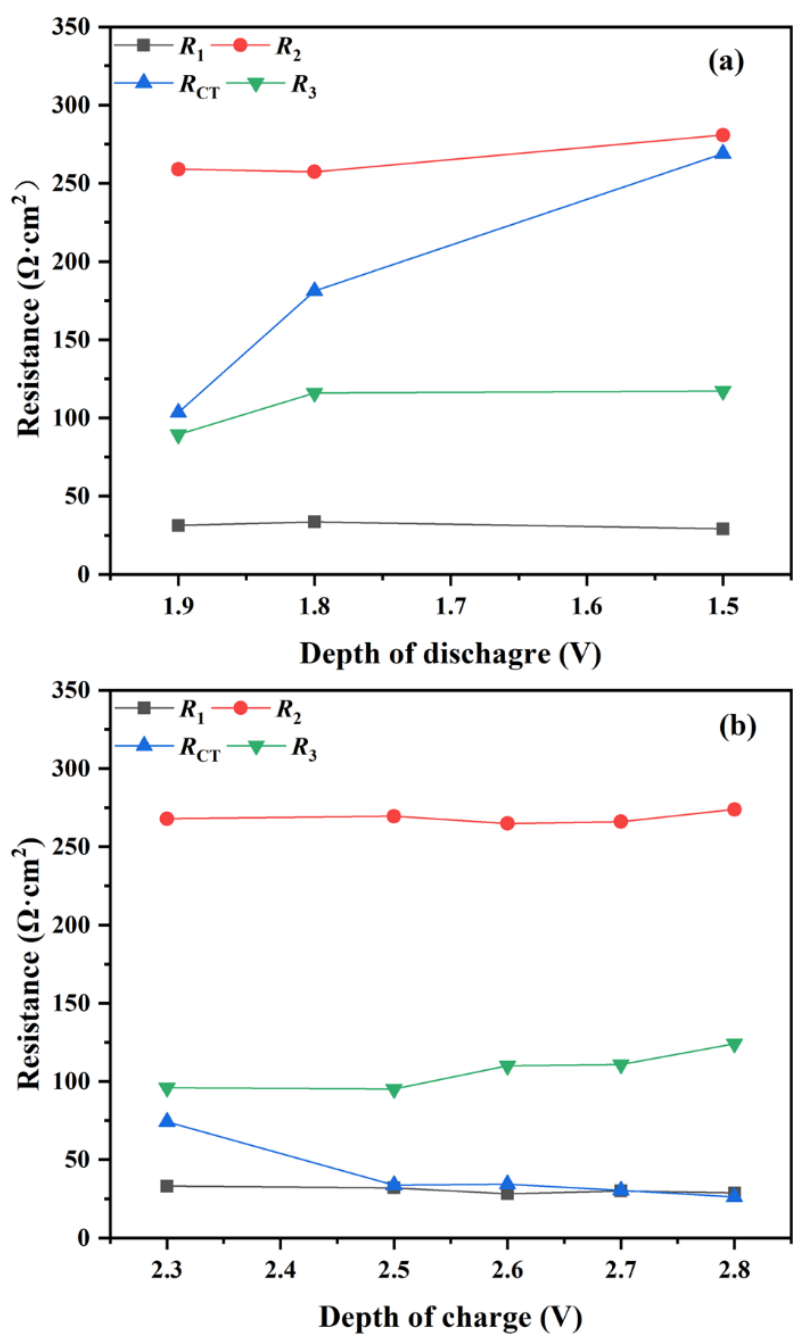

Figure 7. Change of the resistances during (a) discharge and (b) charge.

Notably, it can be seen in Figure 5 that the change of the middle-frequency semicircle with the depth of discharge is very obvious. As discharging progresses, the middle frequency semicircle gradually enlarges. The mid-frequency semicircle is typically attributed to the charge transfer resistance at the cathode and the double layer capacitance formed at the corresponding interface.[ 29, 31-34] The variation of the charge transfer resistance $R_{\mathrm{CT}}$ during a cycle is clearly illustrated in Figure 7 . During discharge $R_{\mathrm{CT}}$ increases from $103 \Omega \cdot \mathrm{cm}^{2}$ to $268 \Omega \cdot \mathrm{cm}^{2}$, while during charge $R_{\mathrm{CT}}$ decreases from $74 \Omega \cdot \mathrm{cm}^{2}$ to $27 \Omega \cdot \mathrm{cm}^{2}$. It indicates that the charge transfer in the cathode becomes difficult when the conversion from $\mathrm{S}$ to $\mathrm{Li}_{\mathrm{x}} \mathrm{S}$ occurs. It has been

\section{ACCEPTED MANUSCRIPT}


widely reported that the charge transfer resistance in solid-state cathode is closed associated with the extent of contact between electrode and solid electrolyte. Yao et al. revealed that the change of charge transfer resistance is closely related to the volume change of the cathode material which changes the contact area between the active materials and the electrolyte.[32] Zhang et al. also proposed that the charge transfer resistance can reflect whether a good electronic/ion conductive network is built in the battery, and that a good network has a great relationship with the interface contact between the electrode and the electrolyte.[33] During discharging, elemental S converts into $\mathrm{Li}_{\mathrm{x}} \mathrm{S}$, leading to $80 \%$ volume expansion of active materials, which changes the stress/strain status in the composite solid cathode and deteriorates the contact between the active material and the solid electrolyte. It results in the evident increase of $R_{\mathrm{CT}}$.

A depressed third semicircle $\left(R_{3} / \mathrm{CPE}_{3}\right)$ is recognized in the low frequency region. According to the EIS fitting, $R_{3}$ is about $90-120 \Omega \cdot \mathrm{cm}^{2}$. Moreover, based on the equation $C=T^{\frac{1}{P}} R^{\frac{1}{P}-1}$, [35] where $T$ and $P$ are the fitting parameters of CPE and $R$ is the parallel resistance, the average capacitance is calculated to be about $10^{-3} \mathrm{~F} \cdot \mathrm{cm}^{-2}$. The capacitance approaches the double-layer capacitance of Li-In/solid electrolyte interface $\left(9.6-1.7 \mathrm{mF} \cdot \mathrm{cm}^{-2}\right),[36]$ we therefore propose that the low-frequency semicircle is assigned to the charge transfer resistance and the related double-layer capacitance at the lithium anode. $R_{3}$ slightly increases from the begin of discharging to the end of charging, which may be attributed to the roughening of the $\mathrm{Li} /$ solid electrolyte interphase by lithium striping and plating.[37]

A $45^{\circ}$ straight line can be observed in the low frequency region, which corresponds

\section{ACCEPTED MANUSCRIPT}


to a Warburg impedance. Moreover, it is found that the slope of the tail at the lowest frequency turning away from $45^{\circ}$, which is described by an intercalation capacitance in the equivalent circuit. This phenomenon is attributed to the accumulation of the Li-ion in the active materials.[26, 27, 38]

Here the Warburg impedance is assigned to the Li diffusion in the battery. The validity of this assignment will soon be discussed. Then the apparent diffusion coefficient $D_{\text {app }}$ can be calculated with the following expression:[26, 27]

$$
D_{\text {app }}=\frac{\left(\frac{Q_{\mathrm{I}} M_{\mathrm{W}} L}{n F C_{\mathrm{L}} \sigma}\right)^{2}}{2}
$$

where $Q_{\mathrm{I}}$ is the specific capacity of the cathode $\left(Q_{\mathrm{I}}=1675 \mathrm{mAh} \cdot \mathrm{g}^{-1}\right), M_{\mathrm{w}}$ is the molecular weight of active materials, $L$ is the length of diffusion path (here use the particle size of about $10 \mathrm{~nm}), n$ is the charge number of $\mathrm{Li}^{+}(n=1), F$ is Faraday constant, $C_{\mathrm{L}}$ is the intercalation capacitance and $\sigma$ is the Warburg coefficient which is estimated to be the reciprocal of W-T in Table 1 and Table 2.

According to the above expression, $D_{\text {app }}$ at different depths of discharge and charge is calculated and illustrated in Figure 8. $D_{\text {app }}$ is in the order of $10^{-19}-10^{-17} \mathrm{~cm}^{2} \cdot \mathrm{s}^{-1}$. First, the diffusion coefficients of $\mathrm{Li}$ ion of $\mathrm{Li}_{7} \mathrm{P}_{3} \mathrm{~S}_{11}\left(\sim 10^{-7} \mathrm{~cm}^{2} \cdot \mathrm{s}^{-1}\right)[39,40]$ and $\mathrm{Li}_{3} \mathrm{PS}_{4}\left(\sim 10^{-}\right.$ $\left.{ }^{9} \mathrm{~cm}^{2} \cdot \mathrm{s}^{-1}\right)[41]$ is much higher than the obtained apparent diffusion coefficient, implying that the diffusion of Li ion is not limited by the diffusion in the electrolyte. Second, the obtained $D_{\text {app }}$ approaches the theoretically calculated diffusion coefficient of $\mathrm{Li}_{\mathrm{x}} \mathrm{S}$.[42] Moreover, the variation of $D_{\text {app }}$ with the lithiation degree follows the predicted trends, that is, first decreases and then increases. Therefore, the Warburg impedance in the EISs can be reasonably assigned to the Li diffusion in the active material $\mathrm{Li}_{\mathrm{x}} \mathrm{S}$. It is worth

\section{ACCEPTED MANUSCRIPT}


noting that the Warburg tail in the EIS of ASSLSBs is much more evident than that of liquid lithium-sulfur batteries.[8,9] This coincides with the fact that the electrochemical reaction in liquid lithium-sulfur batteries is mediated by the diffusion of soluble polysulfide ions in the electrolyte which is much faster than the Li diffusion in the solid active materials. Considering that the diffusion-associated Warburg impedance prevails the EIS of the studied ASSLSBs, we infer that the main factor limiting the reaction kinetics of the ASSLSB is the diffusion of Li in the cathode active materials.

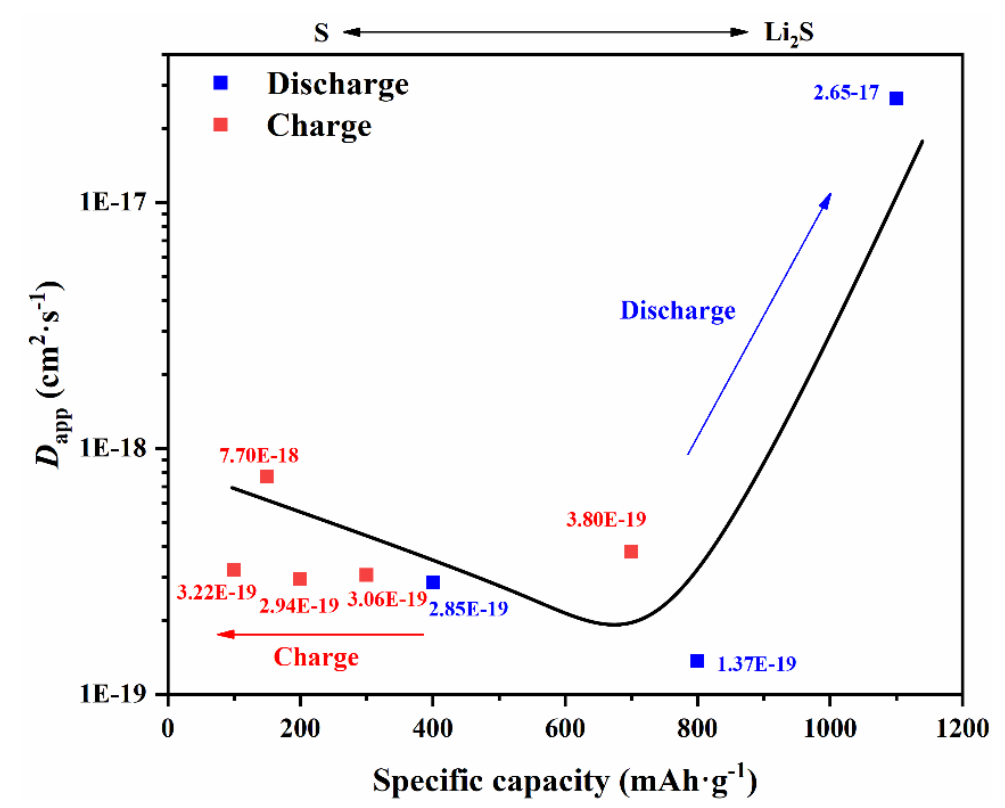

Figure 8. Apparent diffusion coefficient of the ASSLSB at different depths of charge/discharge.
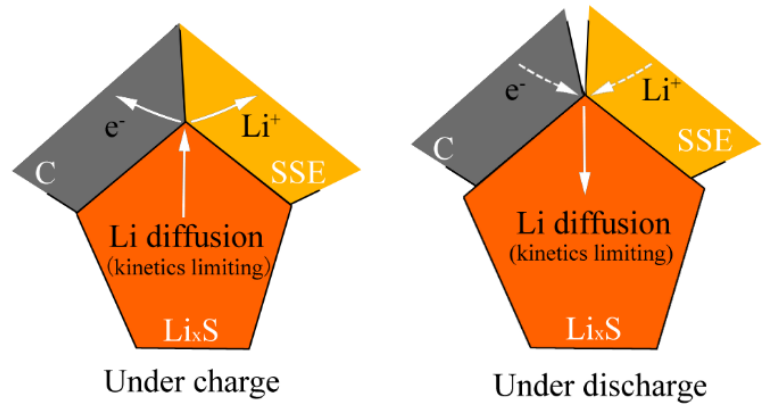

Figure 9. Proposed mechanism model for the electrochemical processes of ASSLSBs.

Based on the quantitative EIS analysis, a mechanism model is proposed for the 
electrochemical processes of ASSLSBs as shown in Figure 9. During charge, Li is extracted from the active material $\mathrm{Li}_{\mathrm{x}} \mathrm{S}$ by the charge transfer at the surface of $\mathrm{Li}_{\mathrm{x}} \mathrm{S}$, and the formed Li ions and electrons transport out of the composite cathode through the ionic channels formed by the solid electrolyte and the electronic channels formed by the conductive agent $\mathrm{KB}$, respectively. Meanwhile, the extraction of Li lowers the its concentration at the surface of $\mathrm{Li}_{\mathrm{x}} \mathrm{S}$. To maintain the current flow, Li should be replenished by the Li diffusion from the internal of $\mathrm{Li}_{\mathrm{x}} \mathrm{S}$ to the surface. Unfortunately, the extremely low diffusion coefficient of $\mathrm{Li}_{\mathrm{x}} \mathrm{S}$ hinders a fast diffusion process, thus limiting the electrochemical reaction kinetics of ASSLSBs.

During discharge, the volume of the active materials expands drastically. The electrolyte and the conductive agent cannot accommodate the volume expansion of the active materials, and the channels of ions and electrons breaks to some extent, leading to the increasing of the charge transfer resistance in the cathode. Moreover, compared the Nyquist plots of charge with that of discharge, it can be found that the tail at the lowest frequency is steeper during discharge. This is because Li-ion apparent diffusion coefficient increases evidently during discharge, and the Warburg impedance becomes smaller. This causes the greater capacitive impedance contribution from the intercalation capacitance.

\section{Conclusions}

In summary, the electrochemical impedance spectra of the ASSLSB at different depths of charge and discharge are characterized, which are well described by a reasonable equivalent circuit. With this equivalent circuit, the evolution of the key

\section{ACCEPTED MANUSCRIPT}


parameters associated with the electrochemical processes, such as the resistance of the electrode/electrolyte interface, the charge transfer resistance of anode and cathode and the Li apparent diffusion coefficient can be monitored. It is revealed that the transfer resistance of cathode evidently increases during discharge, which is ascribed to the partial broken of the ionic and electronic channels in the composite cathode due to the volume expansion of $\mathrm{Li}_{\mathrm{x}} \mathrm{S}$. It is also found that the apparent diffusion coefficient is in the order of $10^{-19}-10^{-17} \mathrm{~cm}^{2} \cdot \mathrm{s}^{-1}$, corresponding to the Li diffusion in $\mathrm{Li} \times \mathrm{S}$. Such as low diffusion coefficient makes the Li diffusion in the active materials the principal kinetics-limiting process in ASSLSBs. Based on these conclusions, several strategies can be used to improve the electrochemical performance of ASSLSBs: (1) Reduce the particle size of the active materials to shorten the diffusion distance of Li. (2) Improve the Li diffusion coefficient of $\mathrm{Li}_{\mathrm{x}} \mathrm{S}$ by doping certain elements. (3) Design novel microstructures of the composite cathode to ameliorate the contact issues arisen by the giant volume change of $\mathrm{Li}_{\mathrm{x}} \mathrm{S}$.

\section{Acknowledgement}

The work is financially supported by the National Natural Science Foundation of China (NFSC) project (No. 51702216), the Natural Science Foundation of Guangdong Province (No. 2021A1515011725) and State Key Laboratory of Silicon Materials Visiting Scholar Fund (SKL2020-10).

\section{References}

[1] S. Li, D. Leng, W. Li, L. Qie, Z. Dong, Z. Cheng, Z. Fan, Energy Storage Materials 27 (2020) 279.

[2] L.P. Hou, H. Yuan, C.Z. Zhao, L. Xu, G.L. Zhu, H.X. Nan, X.B. Cheng, Q.B. Liu, C.X. He, J.Q. Huang, Q. Zhang, Energy Storage Materials 25 (2020) 436.

[3] J. Yi, L. Chen, Y. Liu, H. Geng, L.Z. Fan, ACS Appl. Mater. Interfaces 11 (2019) 36774.

\section{ACCEPTED MANUSCRIPT}


[4] Z. Fan, B. Ding, T. Zhang, Q. Lin, V. Malgras, J. Wang, H. Dou, X. Zhang, Y. Yamauchi, Small 15 (2019) 1903952.

[5] H. Kim, J.T. Lee, A. Magasinski, K. Zhao, Y. Liu, G. Yushin, Adv. Energy Mater. 5 (2015) (24) 1501306.

[6] Z. Yang, Z. Zhu, J. Ma, D. Xiao, X. Kui, Y. Yao, R. Yu, X. Wei, L. Gu, Y. S. Hu, H. Li, X. Zhang, Adv. Energy Mater. 6 (2016) 1600806.

[7] S. Wenzel, S. Randau, T. Leichtweiß, D.A. Weber, J. Sann, W.G. Zeier, J. Janek, Chem. Mater. 28 (2016) 2400.

[8] Z. Deng, Z. Zhang, Y. Lai, J. Liu, J. Li, Y. Liu, J. Electrochem. Soc. 160 (2013) A553.

[9] N.A. Cañas, K. Hirose, B. Pascucci, N. Wagner, K.A. Friedrich, R. Hiesgen, Electrochim. Acta 97 (2013) 42.

[10] H. Jiang, Y. Han, H. Wang, Y. Zhu, Q. Guo, H. Jiang, C. Zheng, K. Xie, Ionics 26 (2020) 4257.

[11] G.H. Chang, Y.S. Oh, S. Kang, J. Y. Park, H.-T. Lim, Electrochim. Acta 358 (2020) 136884.

[12] Y. Wang, T. Liu, L. Estevez, J. Kumar, Energy Storage Materials 27 (2020) 232.

[13] B. Fan, Y. Xu, R. Ma, Z. Luo, F. Wang, X. Zhang, H. Ma, P. Fan, B. Xue, W. Han, ACS Appl. Mater. Interfaces 12 (2020) 52845.

[14] Y. Lu, S. Gu, X. Hong, K. Rui, X. Huang, J. Jin, C. Chen, J. Yang, Z. Wen, Energy Storage Materials 11 (2018) 16.

[15] J. Liang, X. Li, Y. Zhao, L.V. Goncharova, G. Wang, K.R. Adair, C. Wang, R. Li, Y. Zhu, Y. Qian, L. Zhang, R. Yang, S. Lu, X. Sun, Adv. Mater. 30 (2018) (45) e1804684.

[16] Z. Wang, M. Feng, H. Sun, G. Li, Q. Fu, H. Li, J. Liu, L. Sun, A. Mauger, C.M. Julien, H. Xie, Z. Chen, Nano Energy 59 (2019) 390.

[17] X. Fu, L. Scudiero, W.H. Zhong, J. Mater. Chem. A 7 (2019) 1835.

[18] M. Nagao, Y. Imade, H. Narisawa, T. Kobayashi, R. Watanabe, T. Yokoi, T. Tatsumi, R. Kanno, J. Power Sources 222 (2013) 237.

[19] Z. Liu, W. Fu, E.A. Payzant, X. Yu, Z. Wu, N.J. Dudney, J. Kiggans, K. Hong, A.J. Rondinone, C. Liang, J. Am. Chem. Soc. 135 (2013) 975.

[20] M. Wild, L. O'Neill, T. Zhang, R. Purkayastha, G. Minton, M. Marinescu, G.J. Offer, Energy Environ. Sci. 8 (2015) 3477.

[21] M. Nagao, A. Hayashi, M. Tatsumisago, T. Ichinose, T. Ozaki, Y. Togawa, S. Mori, J. Power Sources 274 (2015) 471.

[22] Z. Wang, Y. Tang, L. Zhang, M. Li, Z. Shan, J. Huang, Small 16 (2020) 2001899.

[23] R. c. Xu, X. h. Xia, X. 1. Wang, Y. Xia, J. p. Tu, J. Mater. Chem. A 5 (2017) 2829.

[24] S. Larfaillou, D. Guy-Bouyssou, F. le Cras, S. Franger, J. Power Sources 319 (2016) 139.

[25] W. Zhang, D.A. Weber, H. Weigand, T. Arlt, I. Manke, D. Schroder, R. Koerver, T. Leichtweiss, P. Hartmann, W.G. Zeier, J. Janek, ACS Appl. Mater. Interfaces 9 (2017) 17835.

[26] M. Mohamedi, A. Makino, K. Dokko, T. Itoh, I. Uchida, Electrochim. Acta 48 (2002) 79.

[27] H. Xia, L. Lu, Phys. Scr. T129 (2007) 43.

[28] Q. Ge, L. Zhou, Y.-m. Lian, X. Zhang, R. Chen, W. Yang, Electrochem. Commun. 97 (2018) 100.

[29] H. Li, T. Zhang, Z. Yang, Y. Shi, Q. Zhuang, Y. Cui, Int. J. Electrochem. Sci. 16 (2021) 210229.

[30] Z. Wu, Z. Xie, A. Yoshida, X. An, Z. Wang, X. Hao, A. Abudula, G. Guan, Chem. Eng. J. 380 (2020) 122419.

[31] A. Sakuda, H. Kitaura, A. Hayashi, K. Tadanaga, M. Tatsumisago, J. Electrochem. Soc. 156 (2009) A27.

\section{ACCEPTED MANUSCRIPT}


[32] X. Yao, N. Huang, F. Han, Q. Zhang, H. Wan, J.P. Mwizerwa, C. Wang, X. Xu, Adv. Energy Mater. 7 (2017) 1602923.

[33] Q. Zhang, H. Wan, G. Liu, Z. Ding, J.P. Mwizerwa, X. Yao, Nano Energy 57 (2019) 771.

[34] S. Ohno, R. Koerver, G. Dewald, C. Rosenbach, P. Titscher, D. Steckermeier, A. Kwade, J. Janek, W.G. Zeier, Chem. Mater. 31 (2019) 2930.

[35] B.X. Bo Fan, Zhongkuan Luo, Xianghua Zhang, Hongli Ma, Laurent Calvez, J. Am. Ceram. Soc. 102 (2019) 1122.

[36] W. Zhang, F.H. Richter, S.P. Culver, T. Leichtweiss, J.G. Lozano, C. Dietrich, P.G. Bruce, W.G. Zeier, J. Janek, ACS Appl. Mater. Interfaces 10 (2018) 22226.

[37] T. Krauskopf, F.H. Richter, W.G. Zeier, J. Janek, Chem. Rev. 120 (2020) 7745.

[38] D. Aurbach, K. Gamolsky, B. Markovsky, G. Salitra, Y. Gofer, U. Heider, R. Oesten, M. Schmidtb, J. Electrochem. Soc. 147 (2000) 1322.

[39] I.H. Chu, H. Nguyen, S. Hy, Y.C. Lin, Z. Wang, Z. Xu, Z. Deng, Y.S. Meng, S.P. Ong, ACS Appl. Mater. Interfaces 8 (2016) 7843.

[40] C. Yu, S. Ganapathy, E.R.H. van Eck, L. van Eijck, N. de Klerk, E.M. Kelder, M. Wagemaker, J. Energy Chem. 38 (2019) 1.

[41] K. Hayamizu, Y. Aihara, T. Watanabe, T. Yamada, S. Ito, N. Machida, Solid State Ionics 285 (2016) 51.

[42] M.M. Islam, A. Ostadhossein, O. Borodin, A.T. Yeates, W.W. Tipton, R.G. Hennig, N. Kumar, A.C. van Duin, Phys. Chem. Chem. Phys. 17 (2015) 3383.

\section{Table and Figure Captions}

Table 1. Fitting parameters of the EISs at different depths of discharge (DOD).

Table 2. Fitting parameters of the EISs at different depths of charge (DOC).

Figure 1. (a) XRD pattern and impedance spectrum of (b) the $\beta-\mathrm{Li}_{3} \mathrm{PS}_{4} / \mathrm{S}$ composite and (c) pure sulfur.

Figure 2. Charge and discharge curves of the (a) ASSLSB and (b) typical liquid lithiumsulfur battery.

Figure 3. Nyquist plots of the ASSLSB at different depths of (a) discharge and (b) charge.

Figure 4. Schematic diagram of the EIS semicircles in different frequency ranges. The insert

\section{ACCEPTED MANUSCRIPT}


shows the proposed equivalent circuit for the EIS of the ASSLSB.

Figure 5. Fitted plots at different depths of discharge: (a) D-1.9 V (b) D-1.8 V (c) D-1.5 V.

Figure 6. Fitted plots at different depths of charge: (a) C-2.3 V (b) C-2.5 V (c) C-2.6 V (d) C$2.7 \mathrm{~V}(\mathrm{e}) \mathrm{C}-2.8 \mathrm{~V}$.

Figure 7. Change of the resistances during (a) discharge and (b) charge.

Figure 8. Apparent diffusion coefficient of the ASSLSB at different depths of charge/discharge.

Figure 9. Proposed mechanism model for the electrochemical processes of ASSLSBs. 